\title{
Low Risk of Developing Chronic Hepatitis E in Heart Transplant Recipients: A Prospective 2-Year Follow-Up Study
}

\author{
Sven Pischke ${ }^{a, d} \quad$ Christoph Bara $^{b}$ Patrick Behrendt ${ }^{a, c} \quad$ Axel Haverich $^{b} \quad$ Michael P. Manns $^{a}$ \\ Heiner Wedemeyer ${ }^{\mathrm{a}}$ \\ ${ }^{a}$ Gastroenterology, Hepatology and Endocrinology, and ${ }^{b}$ Cardiovascular, Thoracic and Transplant Surgery, \\ Hannover Medical School, and ' Division of Experimental Virology, TWINCORE, Center for Experimental and \\ Clinical Infection Research, Hannover, and d Department of Internal Medicine, University Medical Center \\ Hamburg-Eppendorf, Hamburg, Germany
}

Dear Editor,

Chronic hepatitis E virus (HEV) infections can cause life-threatening consequences in immunosuppressed patients, especially in heart transplant recipients (HTR) [1]. A reduction of immunosuppression or ribavirin treatment is a therapeutic strategy [1]. Anti-HEV seroprevalence rates are higher in HTR (11\%) and nontransplant cardiac patients $(7 \%)$ compared to healthy controls (2\%), as tested by the anti-HEV MP assay (MP Biomedicals, Singapore) [1].

However, a novel widely used antiHEV assay, the Wantai assay (Wantai, Beijing, China) has a higher specificity (99.6\%) and sensitivity (98\%) [2,3], especially in immunosuppressed individuals [4]. Anti$\mathrm{HEV}$ frequencies of $30 \%$ were detected by the Wantai assay in the general German population [5], while only $2-4.5 \%$ tested anti-HEV positive by the MP assay $[1,5]$.

The aims of this study were as follows: 1 to compare the Wantai assay and the MP assay in 225 HTR;
2 to determine the risk of de novo chronic HEV infection in Germany by PCR [1] within a 2-year follow-up cohort of HTR; and

3 to compare the previous and recent anti-HEV MP results in a subcohort of 201 patients who had been tested 2 years previously [1].

Therefore, we tested all the 225 HTR by PCR for the presence of HEV and by the $\mathrm{MP}$ and the Wantai IgG assays for the presence of HEV-specific antibodies (Fig. 1).

The Wantai assay tested more frequently positive for anti-HEV IgG than the MP assay ( 42 vs. $6 \%, p=0.001, \chi^{2}$ test; Fig. 1). Twelve patients (5\%) tested positive by both assays, 83 (37\%) were Wantai positive only and 2 patients (1\%) showed MPpositive/Wantai-negative results. The finding of $42 \%$ anti-HEV positivity (Wantai) in HTR was significantly higher than in a healthy German cohort [5] (30\%, $p=0.007$, $\chi^{2}$ test), which is remarkable, as antibody levels are usually lower in highly immuno- suppressed patients. Recently, a study on HIV-infected patients in Germany has determined an anti-HEV seroprevalence rate of $26 \%$ for the Wantai test and $1.6 \%$ for the MP assay [6], which is much lower than in the present study on HTR $(p=0.01, p=$ $0.0001)$. Thus, our study confirms the higher risk of HEV exposure in HTR, as previously reported with the MP assay [1]. This study does not explain this observation. However, we assume that frequent blood products received by HTR might be a possible explanation for the increased risk of HEV exposure.

Similar to previous studies, patient age was higher in Wantai-positive HTR (mean 63 vs. 54 years, $p<0.001$, Mann-Whitney test), but anti-HEV positivity was not associated with gender or underlying heart disease.

A previously positive MP assay result could be confirmed in $12 / 18$ patients, indicating that anti-HEV antibody levels decreased in at least one-third of HTR within 2 years of follow-up. Of note, $3 / 6$ patients

\section{KARGER}

(C) 2017 S. Karger AG, Basel

0300-5526/17/0596-0254\$39.50/0

E-Mail karger@karger.com

www.karger.com/int
Prof. Dr. Heiner Wedemeyer

Gastroenterology, Hepatology and Endocrinology Hannover Medical School, Carl-Neuberg-Strasse 1 DE-30625 Hannover (Germany)

E-Mail wedemeyer.heiner@mh-hannover.de 
Fig. 1. Test results for Wantai and MP assays. ULN, upper limit of normal.

who became MP negative still tested Wantai positive, indicating a higher sensitivity of this assay.

In the initial study, patients with chronic HEV infection who cleared the infection by ribavirin therapy were reported [1]. Interestingly, anti-HEV was lost after HEV clearance in $1 / 2$ patients where follow-up MP testing was possible. Both patients remained Wantai positive. These findings highlight the limitations of the MP assay and the value of the Wantai test in immunosuppressed individuals.

\section{References}

1 Pischke S, Stiefel P, Franz B, Bremer B, Suneetha PV, Heim A, Ganzenmueller T, Schlue J, Horn-Wichmann R, Raupach R, Darnedde M, Scheibner Y, Taubert R, Haverich $\mathrm{A}$, Manns MP, Wedemeyer $\mathrm{H}$, Bara CL: Chronic hepatitis E in heart transplant recipients. Am J Transplant 2012;12:3128-3133.

2 Bendall R, Ellis V, Ijaz S, Ali R, Dalton H: A comparison of two commercially available anti-HEV IgG kits and a re-evaluation of antiHEV IgG seroprevalence data in developed countries. J Med Virol 2010;82:799-805.

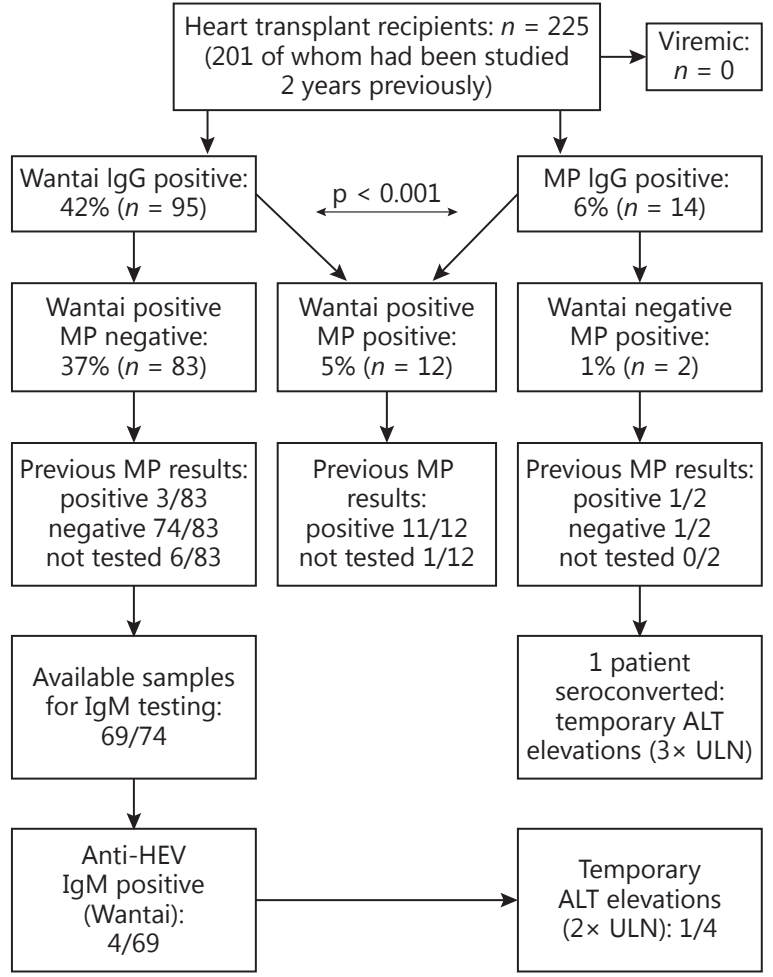

De novo anti-HEV seroconversion with the MP assay was observed in 1 patient within 2 years of follow-up. This was not confirmed by the Wantai assay, indicating false positivity. No new persistent HEV infection was identified in any HTR during 2 years of follow-up, while $2 \%$ of HTR had previous chronic HEV infection [1]. Thus, the annual risk of developing chronic HEV infection in German HTR is below $0.25 \%$.

3 Yan Q, Du H, Wang Y, Ge S, Huang S, Jiang L, Zhang J, Xia NS: Comparison of two diagnostic reagents to detect anti-hepatitis $E$ virus IgG antibodies. Chin J Zoonoses 2008;24: 1087-1089.

4 Rossi-Tamisier M, Moal V, Gerolami R, Colson P: Discrepancy between anti-hepatitis $\mathrm{E}$ virus immunoglobulin $G$ prevalence assessed by two assays in kidney and liver transplant recipients. J Clin Virol 2013;56:62-64.

\section{Conclusions}

More than $40 \%$ of HTR have serological evidence of previous HEV contact, which is higher than in healthy individuals or HIVinfected patients. HEV transmission by blood products is one possible explanation for this observation.

The risk of chronic HEV infection in HTR should not be overestimated in Central Europe. Screening for HEV RNA in patients with elevated transaminases seems to be sufficient.

5 Wenzel JJ, Preiss J, Schemmerer M, Huber B Jilg W: Test performance characteristics of Anti-HEV IgG assays strongly influence hepatitis E seroprevalence estimates. J Infect Dis 2013;207:497-500

6 Pischke S, Schwarze-Zander C, Bremer B, Lehmann P, Wiegand SB, Gisa A, Behrendt B, Strassburg CP, Manns MP, Wedemeyer H, Rockstroh J: Hepatitis E virus seroprevalence rate in HIV-infected patients in Germany: a comparison of two commercial assays. Intervirology 2015;58:283-287. 